\title{
INTEGRAL COHOMOLOGY AND DETECTION OF $w$-BASIC 2-GROUPS
}

\author{
KIMBERLY PEARSON
}

\begin{abstract}
In this paper we compute the cohomology $H^{*}\left(G ; Z^{w}\right)$ of all $w$ basic 2-groups $(G, w)$ with integral coefficients twisted by the orientation character $w$. We also calculate appropriate restiction maps and thus prove that the cohomology of any $w$-basic group is detected by subgroups isomorphic to one of five types, and we provide a sample application of this main theorem.
\end{abstract}

\section{INTRODUCTION}

The $w$-basic 2 -groups were introduced and classified by Hambleton, Taylor, and Williams [7], who demonstated the fundamental role of these groups in quadratic representation theory. These groups provide the inductive building blocks for quadratic forms over group rings $Q G$, given an involution induced by $w$.

The $w$-basic groups include some familiar groups, and the cohomology of several of these groups has been studied before. The cohomology of the semidihedral group was computed by Evens and Priddy [6]; the mod-2 cohomology of the metacyclic 2-groups has been done by Diethelm [5], Munkholm [11], and Rusin [13]. The cohomology of all $w$-basic groups with mod-2 coefficients and in some cases with integral coefficients has been computed by Davis and Milgram [4]; Rusin's work on the cohomology of groups of order 32 [14] is also applicable as each family of $w$-basic groups has a representative of order thirty-two.

The results of this paper are applied by Davis in [3] to classify equivariant intersection forms arising from closed manifolds which are the total space of a finite $G$-cover. These forms are analyzed by characteristic class formulae involving higher-index homomorphisms whose domain is the homology of $G$. Quadratic representation theory [7] gives a reduction to the homology of $w$-basic 2-groups, where an explicit analysis is required. In addition, the computation of the homology of $w$-basic 2-groups would be a necessary tool for an attempt to generalize the work of Milgram, Hambleton, Taylor, and Williams on surgery with finite fundamental group in [10] and [8] to the case of nonoriented manifolds, and hence would play a key role in any systematic classification of nonoriented manifolds with finite fundamental group.

\section{MAin RESUlt}

Let $G$ be a finite 2-group. The group $G$ is basic if its abelian normal subgroups are cyclic. If $w$ is a homomorphism from $G$ to $Z_{2}$, then the pair $(G, w)$ is $w$-basic if

Received by the editor November 17, 1993 and, in revised form, February 11, 1995.

1991 Mathematics Subject Classification. Primary $20 J 06$. 
all abelian normal subgroups of $G$ that are contained in the kernel of $w$ are cyclic. Note that if $G$ is basic, then $G$ is $w$-basic for all $w$. These basic and $w$-basic groups are classified in [7] and are listed with presentations here.

The four families of basic groups are quite familiar:

cyclic groups, $Z_{2^{i}}$;

dihedral groups, $D_{2^{i+1}}=\left\langle x, y \mid x^{2^{i}}=y^{2}=1, y x y^{-1}=x^{-1}\right\rangle, i \geq 3$;

quaternionic groups, $Q_{2^{i+1}}=\left\langle x, y \mid x^{2^{i-1}}=y^{2}, y^{4}=1, y x y^{-1}=x^{-1}\right\rangle, i \geq 2$;

semidihedral groups, $S D_{2^{i+1}}=\left\langle x, y \mid x^{2^{i}}=y^{2}=1, y x y^{-1}=x^{-1} x^{2^{i-1}}\right\rangle, i \geq 3$.

Lemma 1 [7]. The pair $(G, w)$ is $w$-basic if and only if $G$ is basic or $G$ is given as a semidirect product

$$
1 \rightarrow \operatorname{ker}(w) \rightarrow G \stackrel{w}{\longrightarrow} Z_{2} \rightarrow 1
$$

where $\operatorname{ker}(w)$ is basic and the twisting is determined by a homomorphism $f: \operatorname{ker}(w)$ $\mapsto Z_{2}$ so that if $f(g)=1$, then $z g z^{-1}=g c$, where $c$ is the unique central element of order 2 in $\operatorname{ker}(w)$ and $z$ generates $Z_{2}$, and if $f(g)=0$, then $z g z^{-1}=g$.

After accounting for isomorphisms between several groups of this form, see [4], the $w$-basic groups can be listed as follows:

1. $(G, w)$, where $G$ is basic and $w$ is any map to $Z_{2}$;

2. $D_{2^{i+1}, f_{1}}=\langle x, y, z| x^{2^{i}}=y^{2}=z^{2}=1, y x y^{-1}=x^{-1}, z x z^{-1}=x x^{2^{i-1}}, z y z^{-1}$ $=y\rangle$, with $w: x, y \mapsto 0, z \mapsto 1$ or $w: x \mapsto 0 ; y, z \mapsto 1$;

3. $D_{2^{i+1}, f_{2}}=\langle x, y, z| x^{2^{i}}=y^{2}=z^{2}=1, y x y^{-1}=x^{-1}, z x z^{-1}=x, z y z^{-1}=$ $\left.y x^{2^{i-1}}\right\rangle$, with $w: x, y \mapsto 0, z \mapsto 1$ or $w: x \mapsto 0 ; y, z \mapsto 1$ or $w: x, z \mapsto 1, y \mapsto 0$;

4. $S D_{2^{i+1}, f_{3}}=\langle x, y, z| x^{2^{i}}=y^{2}=z^{2}=1, y x y^{-1}=x^{-1} x^{2^{i-1}}, z x z^{-1}=x x^{2^{i-1}}$, $\left.z y z^{-1}=y x^{2^{i-1}}\right\rangle$, with $w: x, y \mapsto 0, z \mapsto 1$ or $w: x \mapsto 0 ; y, z \mapsto 1$;

5. $W_{2^{i+1}}=Z_{2^{i}, f_{1}}=\left\langle x, y \mid x^{2^{i}}=y^{2}=1, y x y^{-1}=x x^{2^{i-1}}\right\rangle$, with $w: x \mapsto 0$, $y \mapsto 1$;

6. $\left(G \times Z_{2}, w\right)$, where $G$ is basic and $\operatorname{ker}(w)=G$.

Definition. For any group $G$, we say the cohomology of $G$ is detected by the subgroups $H_{1}, H_{2}, \ldots, H_{n}$ if

$$
\bigoplus r_{i}^{*}: H^{*}(G) \rightarrow \oplus H^{*}\left(H_{i}\right)
$$

is injective, where $1 \leq i \leq n$ and $r_{i}^{*}$ is the restriction.

The philosophy is that if the cohomology of $G$ is detected, and if we understand the cohomology of the subgroups and know the restriction maps, then we understand the cohomology of the larger group. For example, if the ring relations or Steenrod squares of the detecting subgroups are known, then we can find the corresponding information for the larger group. Davis and Milgram prove the following detection theorem for $F_{2}$ cohomology of $w$-basic groups.

Theorem [4]. Let $(G, w)$ be a w-basic 2-group. Then for all $j, H^{j}\left(G ; F_{2}\right)$ is detected by subgroups isomorphic to the following five types:

1. abelian groups;

2. generalized quaternionic groups;

3. $Q_{2^{i}} \times Z_{2}$; 
4. $W_{2^{i+1}}$;

5. $S D_{2^{i+1}, f_{3}}$.

In particular, for $j \equiv 0 \bmod 4$ one only needs types $1,3,5$; for $j \equiv 1 \bmod 4$ one only needs types 1,3 ; for $j \equiv 2 \bmod 4$ one only needs types $1,2,3,4$; and for $j \equiv 3$ mod 4 one only needs types $1,2,3$.

The cohomology $H^{*}\left(G ; F_{2}\right)$ and the restriction maps needed to obtain these results are recalled in the Appendix; there, we correct the theorem as stated in [4] by showing that $S D_{2^{i+1}, f_{3}}$ is needed in degrees congruent to $0 \bmod 4$.

Given a $w$-basic group $(G, w)$, let $G$ act on the integers via $g \cdot n=n$ if $w(g)=0$ and $g \cdot n=-n$ if $w(g)=1$. We call the resulting $G$-module $Z^{w}$, and consider the cohomology $H^{*}\left(G ; Z^{w}\right)$. Detection in the integral case is quite different from detection in the mod-2 case.

In this paper we prove the following theorem.

Theorem. Let $(G, w)$ be a w-basic 2-group. Then for all $j, H^{j}\left(G ; Z^{w}\right)$ is detected by subgroups isomorphic to the following five types:

1. abelian groups;

2. generalized quaternionic groups;

3. $Q_{2^{i}} \times Z_{2}$;

4. semidihedral groups;

5. $S D_{2^{i+1}, f_{3}}$.

In particular, for $j \equiv 0 \bmod 4$ one only needs types $1,2,3,5$; for $j \equiv 1 \bmod 4$ one only needs types 1,3 ; for $j \equiv 2 \bmod 4$ one only needs types 1,3 ; and for $j \equiv 3 \bmod$ 4 one only needs types $1,2,3,4$.

The topological motivation for this paper is that charecteristic class formulae for surgery obstructions and for the Mischencko-Ranicki symmetric signature for manifolds with finite fundamental group are given in terms of universal homomorphisms $[16$, Theorems B and C], $[8,1.10],[17]$ whose domains are the homology of the group. Detection theorems [7] allow one to consider only $w$-basic 2 -groups. Rather than review the machinery of characteristic class formulae, we give a sample application of the above computations.

Theorem. Let $f: M \rightarrow N$ be a degree-one normal map, where $N$ is a closed manifold of dimension greater than four with finite fundamental group $G$ and orientation $w=w_{1}: G \rightarrow Z_{2}$. Suppose that for all subquotients $(H / K, w)$ of $(G, w)$ (i.e., $K$ is a normal subgroup of a subgroup $H$ of $G$ with $w$ trivial on $K$ ) of the form:

abelian, quaternionic, $Q \times Z / 2$, or $W$ if $\operatorname{dim} N \equiv 0 \bmod 4$;

abelian, quaternionic, $Q \times Z / 2$ if $\operatorname{dim} N \equiv 1 \bmod 4$;

abelian, $Q \times Z / 2$, semidihedral, or $S D_{f_{3}}$ if $\operatorname{dim} N \equiv 2 \bmod 4$;

or abelian, quaternionic, $Q \times Z / 2, S D_{f_{3}}$ if $\operatorname{dim} N \equiv 3 \bmod 4$,

the map $\tilde{f} / H \times \mathrm{Id}: \tilde{M} / H \times S^{1} \rightarrow \tilde{N} / H \times S^{1}$ is normally bordant to a $Z[H / K]$ homology equivalence. Then $f \times \mathrm{Id}: M \times S^{1} \rightarrow N \times S^{1}$ is normally bordant to a homotopy equivalence.

Proof. By projective surgery theory [12] the above statement is equivalent to the algebraic statement that the surgery obstruction $\sigma(f)$ in $L_{n}^{p}(Z G, w)$ is zero if $\pi_{*} i^{*}(f)$ in $L_{n}^{p}(Z[H / N], w)$ is zero for all such subquotients. The fact that $\sigma(f)$ is zero if restricted to a 2-Sylow subgroup was first noticed by Wall [17]. One further reduces 
to $w$-model groups by the detection theorem $[7,1 . \mathrm{C} .8]$. The cohomology calculations of the theorems above combined with the existence of the characteristic class formula give the result.

Remark. The above is just a sample statement. Much more precise results can be obtained by combining specific computations of $L$-groups with computation of the group homology for particular orientation characters $w$.

To prove the main theorem, we compute the integral cohomology $H^{*}\left(G ; Z^{w}\right)$ of each $w$-basic group $(G, w)$ one at a time. After calculating the appropriate restriction maps, we take inventory and find that the above five families of groups are the ones needed for detection.

As the proof of the theorem is a long sequence of calculations, which can be sometimes repetitive, we begin by describing the general techniques used in computing the cohomology and the restriction maps. We then give a short description of the detection situation for each $w$-basic group. The actual calculations, giving generators and module structure of $H^{*}\left(G ; Z^{w}\right)$ as a module over $H^{*}(G ; Z)$ and full descriptions of the necessary restriction maps, are provided in the Appendix.

The untwisted integral cohomology, i.e., when $w$ is the trivial homomorphism, is well known for the dihedral group and generalized quaternionic groups, and is computed in [6] for the semidihedral group. The $w$-twisted cohomology for many $w$-basic groups is computed in [4]. In the next section, we outline the techniques used to compute the cohomology of the remaining groups.

\section{Calculational techniques}

The Bockstein spectral sequence (BSS) is the principal tool for obtaining the integral cohomology $H^{*}\left(G ; Z^{w}\right)$ from the mod-2 cohomology (see [1]). We recall that it is derived from an exact couple, the $E_{1}$ term is equal to $H^{*}\left(G ; F_{2}\right)$, and the first differential $d_{1}$ is given by $d_{1}(\alpha)=S q^{1}(\alpha)+\alpha \cup W$, where $W=w^{*} x$, with $\langle x\rangle=H^{1}\left(Z_{2} ; F_{2}\right)$. Generators of the image of $d_{1}$ in $H^{j}\left(G ; F_{2}\right)$ represent generators of the $Z_{2}$ summands of $H^{j}\left(G ; Z^{w}\right)$, and generators of the image of $d_{n}$ represent generators of $Z_{2^{n}}$ summands. Elements which live to infinity represent the free part of $H^{*}\left(G ; Z^{w}\right)$, so for $G$ finite the $E_{\infty}$ term will consist of at most a single term in degree zero. In our cases, almost everything dies at $d_{1}$ and the $E_{2}$ term is relatively small.

Our first line of attack on the $E_{2}$ term is to look for a subgroup $H$ of $G$ such that $r^{*}: H^{*}\left(G ; F_{2}\right) \rightarrow H^{*}\left(H ; F_{2}\right)$ gives an isomorphism from $E_{2}(G)$ to $E_{2}(H)$ or to a direct summand of $E_{2}(H)$. If such an isomorphism on $E_{2}$ terms can be found, it induces an isomorphism on $E_{n}$ for all $n$ greater than 2 by the Comparison theorem $[9$, p. 355 , Ch. $11, \S 11]$. If $H^{*}\left(H ; Z^{w}\right)$ is known, we now know the exponent of the elements of higher torsion and we are done. This technique works in many cases if one lets $H$ to be a maximal cyclic subgroup (see [4]).

When no such subgroup can be found, we use the Leray-Serre spectral sequence (LSSS) with twisted integral coefficients in order to determine the exponent of elements in low degrees, perhaps in $H^{2}$ and $H^{3}$. Often we can show that other classes of exponent greater than two arise as products of these low-degree classes, and it turns out that our detection results suffice to determine the exponents of the products.

These methods are sufficient to compute the integral cohomology of all $w$-basic 
groups from their mod-2 cohomology with only one exception, the quaternionic group with trivial coefficients, which is well known anyway.

Once the cohomology has been computed, the next goal is to find the restriction maps. Exploiting the mod-2 results, for each $w$-basic group $(G, w)$, we first compute the integral restrictions for the same subgroups which detected the cohomology of the group with $F_{2}$ coefficients; these mod-2 restriction maps are provided in [4].

Lemma 2. Let $H$ be a subgroup of $G, w: G \rightarrow Z_{2}$, $\bar{w}$ the restriction of $w$ to $H, r:$ $H \rightarrow G$ the inclusion. If $r^{*}: H^{j}\left(G ; F_{2}\right) \rightarrow H^{j}\left(H ; F_{2}\right)$ is injective, then the kernel of $r^{*}: H^{j}\left(G ; Z^{w}\right) \rightarrow H^{j}\left(H ; Z^{\bar{w}}\right)$ at most consists of classes $\left\{\alpha \in H^{j}\left(G ; Z^{w}\right) \mid \alpha=\right.$ $2 \gamma$ for some $\gamma$ \}.

The following diagram gives the proof:

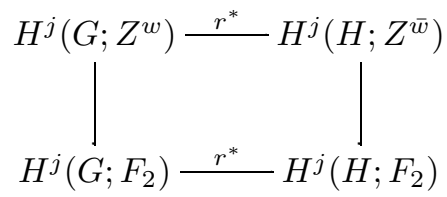

Hence, the only classes of $H^{j}\left(G ; Z^{w}\right)$ which are not detected are either scalar multiples of classes with high exponent, or else reduce mod-2 to an undetected element of $H^{j}\left(G ; F_{2}\right)$. In fact, the above diagram determines $r^{*}$ up to elements of $H^{j}\left(H ; Z^{\bar{w}}\right)$ that are in the image of multiplication by 2 . In particular, if $H^{j}\left(H ; Z^{\bar{w}}\right)$ is all exponent 2, then $r^{*}$ is determined completely.

A second technique is to make use of the ladder of long exact sequences on cohomology coming from the short exact sequence of $G$-modules, $1 \rightarrow Z^{w} \stackrel{\times 2}{\longrightarrow}$ $Z^{w} \rightarrow F_{2} \rightarrow 1$. Here the connecting homomorphism $\beta^{w}$ coincides with $d_{1}$ from the BSS, and all vertical maps are restrictions. We will call this the BLES for short.

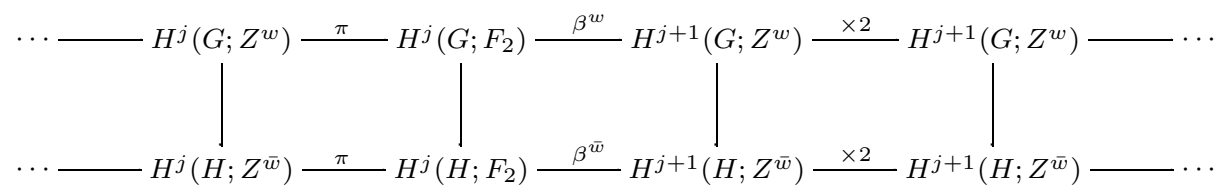

A combination of these two techniques works in almost every case. To handle the few remaining ambiguities, we look at

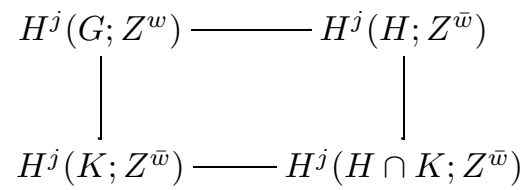

where $K$ is a better-behaved subgroup, all maps are restrictions. Repeating this with several different $K$ 's in stubborn situations, we find enough information to completely determine the integral restriction maps given the mod-2 restriction maps in all cases.

Some $w$-basic groups require more subgroups to detect their integral cohomology than was needed for their mod-2 detection. To compute the additional restriction maps, we compute the maps with $F_{2}$ coefficients first, and then follow our above techniques for obtaining the integral maps out of the mod-2 results. Most of the mod-2 maps we can determine by using the following Gysin sequence and double coset formula. 
Theorem. Let $H$ be an index-two subgroup of $G$, and $\mu$ the unique nontrivial class in $H^{1}\left(G ; F_{2}\right)$ such that the restriction of $\mu$ to $H^{1}\left(H ; F_{2}\right)$ is zero. Then

$$
\cdots \rightarrow H^{i}\left(G ; F_{2}\right) \stackrel{r^{*}}{\longrightarrow} H^{i}\left(H ; F_{2}\right) \stackrel{\operatorname{tr}}{\longrightarrow} H^{i}\left(G ; F_{2}\right) \stackrel{\cup \mu}{\longrightarrow} H^{i+1}\left(G ; F_{2}\right) \stackrel{r^{*}}{\longrightarrow} \cdots
$$

is exact.

We refer the reader to [15] for a proof. We use the following linearity property $[2$, p. 256 , Ch. $12, \S 8]$ for help in computing tr,

$$
\operatorname{tr}\left(\left(r^{*} a\right) \cdot b\right)=a \cdot \operatorname{tr}(b) .
$$

We usually do not need the double coset formula (see [2, Ch. 12]) in its full generality, but use the following "baby" version,

$$
r^{*} \circ \operatorname{tr}(\alpha)=\alpha+c^{*} \alpha,
$$

where $r$ is restriction to an index-two subgroup $H$, and $c$ is conjugation by an element not in $H$.

\section{Summary of Detection Results}

4.1. The dihedral group $D_{2^{i+1}}$ for all $w$ is detected by its mod-2 detecting subgroups, two copies of $Z / 2 \times Z / 2$, and the maximal cyclic subgroup $\langle x\rangle$.

4.2. The integral cohomology of the quaternions, $H^{*}(Q ; Z)$, is detected by smaller quaternionics and eventually copies of $Z_{4}$ except in degrees $\equiv 0 \bmod 4$. The elements $2^{i} \kappa^{n}$ are not detected by any subgroup, where $\kappa$ generates $H^{4}(Q ; Z)$. The twisted cohomology $H^{*}\left(Q ; Z^{w}\right)$ for each nontrivial $w$ is detected by abelians except in degrees $\equiv 3 \bmod 4$. The generator $\alpha$ of $H^{3}\left(Q ; Z^{w}\right)$ for each $w$ is not detected, nor is $\kappa^{n} \cdot \alpha$ in $H^{4 n+3}\left(Q ; Z^{w}\right)$. Though the cohomology of $Q_{8}$ is slightly different than that of the higher-order quaternionics, the detection situation is exactly the same.

4.3. The semidihedral group nicely illustrates the full range of detection possibilities as the homomorphism $w$ is varied. The ring $H^{*}(S D ; Z)$ is detected by its cyclic and dihedral subgroups (hence by abelians); this is an improvement over the mod-2 situation, where a quaternionic subgroup is needed for detection in degrees congruent to $2 \bmod 4$. The cohomology of the $w$-basic group $(S D, w: x \mapsto 0, y \mapsto 1)$ is also detected by abelian subgroups. For $(S D, w: x, y \mapsto 1)$, the quaternionic subgroup is needed for detection in degrees $\equiv 2 \bmod 4$. Finally, $(S D, w: x \mapsto 1, y \mapsto 0)$ is not detected by any proper subgroup in degrees $\equiv 3 \bmod 4$.

4.4. The ring $H^{*}\left(D_{2^{i+1}, f_{2}} ; Z\right)$ is detected by the abelian subgroups which detected $D_{f_{2}}$ with $F_{2}$ coefficients, and the quaternionic subgroup $\langle x, y z\rangle$, which is needed in degrees $\equiv 0 \bmod 4$ to detect some elements of high exponent. The abelian subgroups detect $\left(D_{f_{2}}, w\right)$ for all nontrivial $w$.

4.5. The ring $H^{*}\left(D_{2^{i+1}, f_{1}} ; Z\right)$ is detected by abelian subgroups. Its mod-2 detecting subgroups, $D_{2^{i}, f_{2}}$ and $D_{2^{i}} \times Z_{2}$, and the cyclic group $\langle x\rangle$ detect $H^{*}\left(D_{f_{1}} ; Z\right)$. These three subgroups are then detected by abelians except for one element in $H^{4 n}\left(D_{f_{2}} ; Z\right)$ for each $n$. However, the only classes of $H^{4 n}\left(D_{f_{1}}\right)$ which hit these elements of $H^{4 n}\left(D_{f_{2}}\right)$ are also detected by $\langle x\rangle$. These same three subgroups detect all the $w$-basic groups $\left(D_{f_{1}}, w\right)$. For these $w$, the restriction $\bar{w}$ to $D_{f_{2}}$ is nontriv- 
ial, so $H^{*}\left(D_{f_{2}} ; Z^{\bar{w}}\right)$ is detected by abelians, and so we have abelian detection of $\left(D_{f_{1}}, w\right)$. We comment that the situation is a bit different when $w$ is not one of the $w$-basic homomorphisms. For two of the three non-w-basic maps we do have abelian detection, but for the non- $w$-basic pair $\left(D_{f_{1}}, w: x \mapsto 1, y \mapsto 0\right)$ we also need $\langle x z, y\rangle \cong\left(S D_{2^{i+1}}, \bar{w}\right)$.

4.6. The integral cohomology $H^{*}\left(S D_{2^{i+1}, f_{3}} ; Z\right)$ is detected by $\left\langle x^{2}, x y, z\right\rangle \cong Q_{2^{i}} \times$ $Z_{2},\left\langle x^{2}, y, z\right\rangle \cong D_{2^{i}, f_{2}}$, and $\langle x z, y z\rangle \cong Q_{2^{i+1}}$, even though $H^{*}\left(S D_{f_{3}} ; F_{2}\right)$ is not detected. These same subgroups work for $H^{*}\left(S D_{f_{3}} ; Z^{w}\right)$ for all $w$-basic $w$, except in degrees $\equiv 0 \bmod 4$, where we have classes not detected by any proper subgroup.

4.7. The twisted cohomology module $H^{*}\left(W_{2^{i+1}} ; Z^{w}\right)$ is detected by abelian subgroups for the trivial and the two $w$-basic homomorphisms.

4.8. Finally, we need to consider all groups of the form $G \times Z_{2}$, where $G$ is basic and $w$ is trivial. The only one which is not detected by subgroups already mentioned is $Q_{2^{i}} \times Z_{2}$, and here we have undetected elements in all degrees.

We note here that our main theorem is sharp; undetected elements of $S D_{f_{3}}$ in degrees $\equiv 0 \bmod 4$, of $S D$ in degrees $\equiv 3 \bmod 4$, and of $Q$ in degrees $\equiv 0$ or $3 \bmod$ 4 show that we have given a minimal list of detecting subgroups for the $w$-basic groups.

Remark. After comparing the integral detection theorem with the $F_{2}$-detection theorem, one might wonder about the detection situation with $\left(Z_{2^{k}}\right)^{w}$ coefficients. Neither $F_{2}$ nor integral detection nor both implies $\left(Z_{2^{k}}\right)^{w}$ detection. For example, take $(G, w)$ equal to the semidihedral group of order thirty-two with $w: x \mapsto 0, y \mapsto$ 1. The semidihedral group is detected with mod-2 coefficients and $H^{*}\left(S D ; Z^{w}\right)$ is detected by abelian subgroups. However, one can check that the element $4 e^{2} \in$ $H^{2}\left(S D ;\left(Z_{8}\right)^{w}\right)$, obtained by reducing $4 e^{2} \in H^{2}\left(S D ; Z^{w}\right)$ mod-8, is not detected by any proper subgroup.

\section{ApPEndix: The CALCULATIONS}

All techniques used in calculation are described in the body of the paper. Though we do not specify at each and every turn which method is used, we will point out the computations which required more techniques than average. For each group we give a list of generators of $H^{*}(G ; Z)$ as a ring, and list generators of $H^{*}\left(G ; Z^{w}\right)$ as a module over $H^{*}(G ; Z)$.

The $F_{2}$-cohomology of the $w$-basic groups, and in some cases the integral cohomology has been computed before. We refer the reader to [5, 4, 6, 11, 13, and 14]. For the details on the mod-2 restriction maps, we refer the reader to [4].

We follow the notation of Davis and Milgram for $F_{2}$ cohomology; here are some comments to familiarize the reader. Each nonabelian $w$-basic group except $W$ has a central subgroup of order two with dihedral or dihedral $\times Z_{2}$ quotient; Davis and Milgram use this extension to compute the cohomology in each case. The basis chosen for $H^{1}\left(D_{2^{i}} ; F_{2}\right)$ is $e, f$, where $e(x)=f(x)=f(y)=1, e(y)=0$, since in this basis we have the convenient relation $e \cup f=0$. The class $h$ represents the generator of $H^{1}\left(Z_{2} ; F_{2}\right)$ when the quotient is $D \times Z_{2}$. If we let $\lambda$ generate $H^{1}$ of the central $Z_{2}$ subgroup of $D$, then $\lambda^{4}$ lives to infinity in the spectral sequence of each $w$-basic group except $S D_{2^{i}, f_{3}}$. The class in $H^{4}\left(G ; F_{2}\right)$ which represents $\lambda^{4}$ is called $\kappa$ in each case. Detection shows that cupping with $\kappa$ is injective for 
each $w$-basic group. Furthermore, we find that $\kappa$ is the mod-2 reduction of a class in $H^{4}(G ; Z)$, which in most cases generates all the higher (i.e., greater than two) torsion of $H^{*}(G ; Z)$. Unless otherwise specified, $2 \alpha=0$ for each class $\alpha$, and we will write $H^{*}\left(Z_{2^{i}} \times Z_{2} ; F_{2}\right)=F_{2}[b, c] \otimes \Lambda(a)$, where $\operatorname{dim}(a)=\operatorname{dim}(c)=1, \operatorname{dim}(b)=2$.

We name the integral classes after the classes to which they reduce mod-2. This can sometimes be misleading; for example, there is a indecomposable element in $H^{2}(D ; Z)$ which we name $e^{2}$ because it reduces to the product $e \cup e$ in $H^{2}\left(D ; F_{2}\right)$. However, whereas reduction mod-2 is nearly an injection in all our cases, and as we use this constantly to obtain integral information from the mod-2 situation, and as the integral cohomology is computed out of mod-2 in the first place, it has proven most convenient not to rename the integral classes.

\section{A1. The Dihedral Group $D_{2^{i+1}}=\left\langle x, y \mid x^{2^{i}}=y^{2}=1, y x y^{-1}=x^{-1}\right\rangle$}

We have $H^{*}\left(D ; F_{2}\right)=F_{2}[e, f, w] /\langle e \cup f\rangle, \operatorname{dim}(e)=\operatorname{dim}(f)=1, \operatorname{dim}(w)=2$. Detected by $\left\langle x^{2^{i-1}}, y\right\rangle$ and $\left\langle x^{2^{i-1}}, x y\right\rangle$, both copies of $Z_{2} \times Z_{2}$; with both of these subgroups, $r^{*}(w)=a^{2}+a b$ where $a$ dual to $x^{2^{i-1}}$. Here $e(x)=f(x)=f(y)=1$ and $e(y)=0$. Using this basis gives us the convenient relation $e \cup f=0$.

Let $w_{j}$ be a map to $Z_{2}$ for each $j: w_{1}: x \mapsto 0, y \mapsto 1, w_{2}: x \mapsto 1, y \mapsto 0$, $w_{3}: x, y \mapsto 1$.

For the dihedral group, all calculations are routine. Using the BSS and injecting to a maximal cyclic subgroup at the $E(2)$ terms computes the cohomology in each case. Letting $r:\langle x\rangle \rightarrow D$ be inclusion, we have

$$
H^{*}(D ; Z)=Z\left[e^{2}, f^{2},(e+f) w, w^{2}\right], \exp \left(w^{2}\right)^{n}=2^{i} ; r^{*}\left(e^{2}\right)=r^{*}\left(f^{2}\right)=2^{i-1} b,
$$
$r^{*}\left(w^{2}\right)=b^{2}$

$H^{*}\left(D ; Z^{w_{1}}\right)=\langle e+f, w\rangle, \exp (w)=2^{i} ; r^{*}(e+f)=0, r^{*}(w)=b ;$

$H^{*}\left(D ; Z^{w_{2}}\right)=\left\langle e, f^{2}, f w\right\rangle$, all exponent two;

$H^{*}\left(D ; Z^{w_{3}}\right)=\left\langle f, e^{2}, e w\right\rangle$, all exponent two.

Each of these integral cohomologies is detected by $\langle x\rangle$ and the two mod-2 detecting subgroups, two copies of $Z / 2 \times Z / 2$. The restrictions are computed using BLES. For example, $e^{2} \in H^{2}(D ; Z)$ is in the kernel of multiplication by two, so it is in the image of $\beta$, and in fact we know from the BSS that $\beta(e)=e^{2}$. Then $r^{*}\left(e^{2}\right)=r^{*}(\beta(e))=\beta\left(r^{*}(e)\right)=\beta(a)$. Exactness of the lower row tells us that $\beta(a)$ must be $2^{i-1} b$ since $2^{i-1} b$ is in the kernel of multiplication by two. By reducing mod-2, the other restriction maps are clear. The element $w^{2} \in H^{4}(D ; Z)$ generates all higher torsion of $H^{*}(D ; Z)$. The cyclic subgroup $\langle x\rangle$ detects $\left(w^{2}\right)^{n}$ for all $n$, and all integer multiples of them as well. Applying Lemma 2 to account for the classes of exponent two, we see $H^{*}(D ; Z)$ is detected by abelian subgroups.

The situation is similar for $H^{*}\left(D ; Z^{w_{1}}\right)$. The element $w \in H^{2}\left(D ; Z^{w_{1}}\right)$ has high exponent, as does any $\left(w^{2}\right)^{n} \cdot w \in H^{4 n+1}\left(D ; Z^{w_{1}}\right)$. These elements and their integer multiples are also detected by $\langle x\rangle$. For $H^{*}\left(D ; Z^{w_{2}}\right)$ and $H^{*}\left(D ; Z^{w_{3}}\right)$, we can immediately invoke Lemma 2 and conclude that we have detection by abelian subgroups.

All ring and module multiplication is then determined by the abelian detection.

$$
\begin{gathered}
\text { A2. THE QUATERNIONIC GRoups } Q_{2^{i+1}}= \\
\left\langle x, y \mid x^{2^{i-1}}=y^{2}, y^{4}=1, y x y^{-1}=x^{-1}\right\rangle \text {, FOR } i \geq 3
\end{gathered}
$$

We have $H^{*}\left(Q ; F_{2}\right)=F_{2}[a, b, \kappa] /\left\langle a b, a^{3}+b^{3}\right\rangle, \operatorname{dim}(a)=\operatorname{dim}(b)=1, \operatorname{dim}(\kappa)=4$, with $a(x)=b(x)=b(y)=1$, and $a(y)=0$. The element $\kappa$ is detected by the central 
$Z_{2}$, but $a^{2}$ and $b^{2}$ are only detected by copies of $Q_{8}$, and $a^{3}$ is not detected at all. This is clear as $a$ and $b$ restrict either to 0 or to $a+b$ in the smaller quaternionic groups, hence $a^{2}$ and $b^{2}$ will be detected but not $a^{3}$.

The maps $w_{j}$ are as before, $r_{1}:\langle y\rangle \cong Z_{4} \rightarrow Q, r_{2}:\langle x y\rangle \cong Z_{4} \rightarrow Q$, and $r_{3}:\langle x\rangle \rightarrow Q$, and we obtain

$H^{*}(Q ; Z)=Z\left[a^{2}, b^{2}, \kappa\right] /\left(a^{2}\right)^{2}=\left(b^{2}\right)^{2}=a^{2} \cup b^{2}=0$, with $\exp \left(\kappa^{n}\right)=2^{i+1} ;$ $r_{1}^{*}: a^{2} \mapsto 0, b^{2} \mapsto 2 b, \kappa \mapsto b^{2} ; r_{2}^{*}: a^{2} \mapsto 2 b, b^{2} \mapsto 0, \kappa \mapsto b^{2} ; r_{3}^{*}: a^{2} \mapsto 2^{i-1} b, b^{2} \mapsto$ $2^{i-1} b, \kappa \mapsto b^{2}$;

$H^{*}\left(Q ; Z^{w_{1}}\right)=\left\langle a+b, a^{2}+b^{2}\right\rangle$, with $\exp \left(a^{2}+b^{2}\right)=2^{i-1}$, and also $\exp \left(a^{2}+b^{2}\right) \cdot \kappa^{n}=$ $2^{i-1} ; r_{1}^{*}\left(a^{2}+b^{2}\right)=r_{2}^{*}\left(a^{2}+b^{2}\right)=0, r_{3}^{*}\left(a^{2}+b^{2}\right)=2 b ;$

$H^{*}\left(Q ; Z^{w_{2}}\right)=\left\langle a, b^{2}\right\rangle ; r_{1}^{*}\left(b^{2}\right)=2 b, r_{2}^{*}\left(b^{2}\right)=0, r_{3}^{*}\left(b^{2}\right)=0 ;$

$H^{*}\left(Q ; Z^{w_{3}}\right)=\left\langle b, a^{2}\right\rangle ; r_{1}^{*}\left(a^{2}\right)=0, r_{2}^{*}\left(a^{2}\right)=2 b, r_{3}^{*}\left(a^{2}\right)=0$.

The trivial integral cohomology of the quaternions is well known, and for two of the nontrivial twistings the BSS collapses at the $E_{2}$ terms. To find the exponent of $a^{2}+b^{2}$ in $H^{2}\left(Q ; Z^{w_{1}}\right)$, we use the LSSS with $Z^{w}$ coefficients associated with $1 \rightarrow$ $\operatorname{ker}(w) \rightarrow Q \stackrel{w}{\longrightarrow} Z / 2 \rightarrow 1$. We work in homology and find $H_{1}\left(Q ; Z^{w}\right) \cong Z / 2^{i-1}$; applying universal coefficients, we find $\exp \left(a^{2}+b^{2}\right)=2^{i-1}$.

All restriction maps are determined by the BLES. For $j \equiv 2 \bmod 4$ we can see that $H^{j}\left(Q ; Z^{w}\right)$ is detected by the three abelian subgroups; this is an improvement over the $F_{2}$ situation. However, detection has worsened in degrees congruent to 0 $\bmod 4$. The class $\kappa^{n} \in H^{4 n}(Q ; Z)$ has exponent equal to the order of the group, so it is impossible for $2^{i} \kappa$ to be detected by any proper subgroup. The situation has not changed in other degrees. To see that the class $a^{3} \in H^{3}\left(Q ; Z^{w_{2}}\right)$ is not detected at all, consider the BLES. We know $\beta^{w}\left(a^{2}\right)=a^{3}$ from the BSS, and recalling the mod-2 detection, we know $r^{*}\left(a^{2}\right)$ is zero for each abelian subgroup. Thus, $r^{*}\left(a^{3}\right)=r^{*}\left(\beta^{w}\left(a^{2}\right)\right)=\beta^{w}\left(r^{*}\left(a^{2}\right)\right)=$ zero for each abelian subgroup.

All module structure can be determined by detection except $a^{2} \cup b^{2}$ in $H^{4}(Q ; Z)$, and $a^{2} \cup \alpha$ or $b^{2} \cup \alpha$, where $\alpha$ is any degree-one class in the twisted cohomologies. But both of these can be quickly solved. Since $H^{3}\left(Q ; Z^{w}\right) \rightarrow H^{3}\left(Q ; F_{2}\right)$ is injective for all $w$, reduction mod-2 determines the products of degree 3. Finally $a^{2} \cup b^{2}$ is solved by noting that both terms come from the dihedral quotient of $Q: a^{2} \cup b^{2}=$ $\pi^{*}\left(e^{2}\right) \cup \pi^{*}\left(f^{2}\right)=\pi^{*}\left(e^{2} \cup f^{2}\right)=\pi^{*}(0)=0$.

The $F_{2}$-cohomology of $Q_{8}$ is slightly different from that of $Q_{2^{i}}$ for $i>3$, but detection and the integral cohomology is very similar; $H^{*}\left(Q_{8} ; F_{2}\right)=F_{2}\left[a, b, \kappa_{4}\right] /$ $\left\langle a b+a^{2}+b^{2}, a^{2} b+a b^{2}\right\rangle$, with $a, b, \kappa$ as before. We find $H^{*}\left(Q_{8} ; Z\right)=Z\left[a^{2}, b^{2}, \kappa\right]$; the class $4 \kappa$ is not detected by any proper subgroup. The twisted cohomologies are $H^{*}\left(Q_{8} ; Z^{w_{1}}\right)=\left\langle a+b, a^{2}+b^{2}\right\rangle ; H^{*}\left(Q_{8} ; Z^{w_{2}}\right)=\left\langle a, a^{2}\right\rangle ; H^{*}\left(Q_{8} ; Z^{w_{3}}\right)=\left\langle b, b^{2}\right\rangle$; the element $a^{2} b$ which occurs in each nontrivial twisting is not detected by any proper subgroup.

\section{A3. The Semidihedral group $S D_{2^{i+1}}=\left\langle x, y \mid x^{2^{i}}=y^{2}=1, y x y^{-1}=x^{-1} x^{2^{i-1}}\right\rangle$}

We have $H^{*}\left(S D ; F_{2}\right)=F_{2}\left[e, f, e_{3}, \kappa_{4}\right] /\left\langle e f, e e_{3}, e^{3}, e_{3}^{2}+f^{2} \cup \kappa\right\rangle$, with $\operatorname{dim}(e)=$ $\operatorname{dim}(f)=1, \operatorname{dim}\left(e_{3}\right)=3, \operatorname{dim}(\kappa)=4$. The elements $e$ and $f$ come from the dihedral quotient.

Let $r_{1}:\langle x\rangle \rightarrow S D, r_{2}:\left\langle x^{2}, y\right\rangle \cong D_{2^{i}} \rightarrow S D$, and $r_{3}:\left\langle x^{2}, x y\right\rangle \cong Q_{2^{i}} \rightarrow S D$ be the inclusions. Then $r_{2}^{*}: e \mapsto 0, f \mapsto e+f, e_{3} \mapsto(e+f) w, \kappa \mapsto w^{2} ; r_{3}^{*}: e \mapsto$ 
$a+b, f \mapsto 0, e_{3} \mapsto a^{3}, \kappa \mapsto \kappa_{4}$, and these three subgroups detect. Note in particular that $Q$ is needed to detect the element $e^{2}$. The maps $w_{j}$ are as before.

Integrally, we get $H^{*}(S D ; Z)=Z\left[e^{2}, f^{2}, e_{3} f^{2}, \kappa\right]$, with $\exp \left(\kappa^{n}\right)=2^{i} ; \quad r_{1}^{*}$ : $e^{2} \mapsto 2^{i-1} b, f^{2} \mapsto 2^{i-1} b, e_{3} f^{2} \mapsto 0, \kappa \mapsto b^{2} ; r_{2}^{*}: e^{2} \mapsto 0, f^{2} \mapsto e^{2}+f^{2}, e_{3} f^{2} \mapsto$ $\left(e^{3}+f^{3}\right) w, \kappa \mapsto w^{2} ; r_{3}^{*}: e^{2} \mapsto a^{2}+b^{2}, f^{2} \mapsto 0, e_{3} f^{2} \mapsto 0, \kappa \mapsto \kappa$.

Here the exponent of $\kappa$ can be computed by injecting to the maximal cyclic subgroup, also see [6]. Each restriction map given is completely determined by reducing mod-2. In the mod-2 case, the kernel of $r_{1}^{*} \oplus r_{2}^{*}$ was $e^{2} \kappa^{n}$. But with integer coefficients, $r_{1}^{*}$ now picks up these elements; hence the cyclic and dihedral subgroups (therefore abelians) detect $H^{*}(S D ; Z)$.

We find $H^{*}\left(S D ; Z^{w_{1}}\right)=\left\langle e+f, e_{3} f, e^{2}\right\rangle$, with $\exp \left(e^{2}\right)=2^{i-1}$, also $\exp \left(e^{2}\right)$. $\kappa^{n}=2^{i-1} ; \quad r_{1}^{*}: e+f \mapsto 0, e_{3} f \mapsto 2^{i-1} b^{2}, e^{2} \mapsto 2 b ; r_{2}^{*}: e+f \mapsto e+f, e_{3} f \mapsto$ $\left(e^{2}+f^{2}\right) w, e^{2} \mapsto 2 w ; r_{3}^{*}: e+f \mapsto a+b, e_{3} f \mapsto 0, e^{2} \mapsto a^{2}+b^{2}$. To find the exponent of $e^{2}$ we use the LSSS to find that $H_{1}\left(S D ; Z^{w_{1}}\right)$ is $Z / 2^{i-1}$. Calculation of the restrictions $r_{1}^{*}$ and $r_{2}^{*}$ are straightforward using the BLES, except $r_{2}^{*}\left(e^{2}\right)$, which requires considering the intersection $\left\langle x^{2}\right\rangle$ of $\langle x\rangle$ and $\left\langle x^{2}, y\right\rangle$ and then using $r_{1}^{*}$.

The second twisting renders $H^{*}\left(S D ; Z^{w_{2}}\right)=\left\langle e, f^{2}, e_{3}\right\rangle$, with $\exp \left(e_{3}\right)=4 ; r_{1}^{*}$ : $e \mapsto a, f^{2} \mapsto 0, e_{3} \mapsto a b ; r_{2}^{*}: e \mapsto 0, f^{2} \mapsto e^{2}+f^{2}, e_{3} \mapsto(e+f) w ; r_{3}^{*}: e \mapsto$ $a+b, f^{2} \mapsto 0, e_{3} \mapsto a^{3}$.

This time the BSS has $E_{2}$ term consisting of $e^{2}, e_{3}$, and $\kappa$ times these two elements. We use the LSSS to compute $H_{2}\left(S D ; Z^{w_{2}}\right)$. We already know $H_{1}$ and $H_{3}$, as the BSS provided complete information on $H^{2}$ and $H^{4}$. This forces enough of the differentials in the LSSS so that $H_{2}$ only has classes of at most exponent 4. On the other hand, it has at least exponent 4 , else $e_{3}$ would not have lived to $E_{2}$ in the BSS.

Note here that $2 e_{3}$ is not detected at all, the kernel of $\bigoplus r_{j}^{*}$ is $2 e_{3} \kappa^{n}$, thus in our main theorem the semidihedral group is necessary for detection in degrees congruent to $3 \bmod 4$.

The final twisting gives us $H^{*}\left(S D ; Z^{w_{3}}\right)=\left\langle f, e^{2}, e_{3} f\right\rangle$, all exponent two; $r_{1}^{*}$ : $f \mapsto a, e^{2} \mapsto 0, e_{3} f \mapsto 0 ; r_{2}^{*}: f \mapsto e+f, e^{2} \mapsto 0, e_{3} f \mapsto\left(e^{2}+f^{2}\right) w ; r_{3}^{*}: f \mapsto$ $0, e^{2} \mapsto a^{2}+b^{2}, e_{3} f \mapsto 2^{i-1} \kappa$. All restrictions are computed using the BLES, and by Lemma 2 these subgroups detect $H^{*}\left(S D ; Z^{w_{3}}\right)$.

The ring and module structures are almost determined by detection, except for the problem in degrees $\equiv 3 \bmod 4$ for the second twisting. We are lucky, however, because all pairs of generators which multiply to give something in a degree $\equiv 3$ mod 4 come from the dihedral quotient, so we can multiply in the submodule where the product structure is understood. For example, take $e+f \in H^{1}\left(S D ; Z^{w_{1}}\right)$ and $e^{2} \in H^{2}\left(S D ; Z^{w_{3}}\right):(e+f) \cup e^{2}=\pi^{*}(e+f) \cup \pi^{*}\left(e^{2}\right)=\pi^{*}\left(e+f \cup e^{2}\right)=\pi^{*}\left(e^{3}\right)=2 e_{3}$. To see $\pi^{*}\left(e^{3}\right)=2 e_{3}$, take $e^{2} \in H^{2}\left(D ; F_{2}\right)$ and we have $\beta\left(\pi^{*}\left(e^{2}\right)\right)=\beta\left(e^{2}\right)=2 e_{3}$ from the BSS, but on the other hand, $\beta\left(\pi^{*}\left(e^{2}\right)\right)=\pi^{*}\left(\beta\left(e^{2}\right)\right)=\pi^{*}\left(e^{3}\right)$.

The rest of the groups are $w$-basic only for certain nontrivial $w$, but we also compute the trivial integral cohomology, as $H^{*}\left(G ; Z^{w}\right)$ is a module over $H^{*}(G ; Z)$. The following three groups have central extensions $1 \rightarrow Z_{2} \rightarrow G \rightarrow D \times Z_{2} \rightarrow 1$, and the degree-one cohomology classes $e, f$ and $h$ arise from the quotient, with $e, f$ as before and $h$ is dual to $z$, the generator of $Z_{2}$. 
A4. The Group $\begin{aligned} D_{2^{i+1}, f_{2}} & =\langle x, y, z| x^{2^{i}}=y^{2}=z^{2}=1, y x y^{-1}=x^{-1}, \\ z x z^{-1} & \left.=x, z y z^{-1}=y x^{2^{i-1}}\right\rangle\end{aligned}$

We have $H^{*}\left(D_{f_{2}} ; F_{2}\right)=F_{2}[e, f, h, \kappa] /\left\langle e f,(e+f) h^{2}\right\rangle, \operatorname{dim}(\kappa)=4$.

Let $r_{1}:\langle x, z\rangle \cong Z_{2^{i}} \times Z_{2} \rightarrow D_{f_{2}}, r_{2}:\left\langle x^{2^{i-2}} z, y\right\rangle \cong Z_{4} \times Z_{2} \rightarrow D_{f_{2}}$ and $r_{3}:\left\langle x^{2^{i-2}} z, x y\right\rangle \cong Z_{4} \times Z_{2} \rightarrow D_{f_{2}}$ be inclusions.

Lemma. We have $r_{i}^{*}(\kappa)=b^{2}+b c^{2}$ for each $i$.

Proof. For each subgroup, we can observe that on the $E_{2}$ term of the LSSS, $\lambda^{4}$ restricts to $\mu^{4}$, where $\kappa$ represents $\lambda^{4}$ and $b$ represents $\mu^{2}$. Thus we know that on cohomology $\kappa$ restricts to $b^{2}$ plus possibly terms coming from "further down the diagonal".

We begin with $r_{1}^{*}(\kappa)$. The subgroup is invariant under conjugation by $y$, and we will use the condition that $c_{y}^{*}\left(r_{1}^{*}(\kappa)\right)=r_{1}^{*}\left(c_{y}^{*}(\kappa)\right)=r_{1}^{*}(\kappa)$ to restrict the possibilities for $r_{1}^{*}(\kappa)$. The Gysin sequence in this case tells us that $\operatorname{tr}(b)=h^{2}$, so that $r_{1}^{*}(\operatorname{tr}(b))=c^{2}$. By the double coset formula, $c_{y}^{*}(b)=b+c^{2} ;$ it is easy to see $c_{y}^{*}(a)=a$ and $c_{y}^{*}(c)=c$. Since $r_{1}^{*}(\kappa)$ must include a $b^{2}$ term, the conjugation requirement forces $r_{1}^{*}(\kappa)$ to be $b^{2}+b c^{2}$ plus possibly $a c^{3}$ and $c^{4}$ terms. By making an appropriate selection for $\kappa$ we may assume $r_{1}^{*}(\kappa)=b^{2}+b c^{2}$.

The other two calculations are similar. As both other subgroups are invariant under conjugation by $z$, we find $c_{z}^{*}\left(r_{i}^{*}(\kappa)\right)=r_{i}^{*}(\kappa)$ for $i=1$ or 2 , and our task is now to determine the map $c_{z}^{*}$. We do the general case, that is, if $\phi: Z / 2^{i} \times Z / 2 \rightarrow$ $Z / 2^{i} \times Z / 2$ maps $g \rightarrow g$ and $h \rightarrow h g^{i-1}$, then we determine $\phi^{*}$ on cohomology. It is clear that $\phi^{*}(a)=a$ and $\phi^{*}(c)=c$. To find $\phi^{*}(b)$, we look at the Gysin sequence associated with the group $W_{2^{i+2}}$ and its subgroup $\left\langle x^{2}, y\right\rangle \equiv Z / 2^{i} \times Z / 2$, as conjugation by $x$ in this case is the same as $\phi(W$ is covered in more detail in A7). We see from exactness of the sequence that $r^{*}(\operatorname{tr}(b))=c^{2}$, so by the double coset formula, $\phi^{*}(b)=b+c^{2}$. Then as in the $r_{1}$ situation, we find $r_{i}^{*}(\kappa)=b^{2}+b c^{2}$ plus possibly $a c^{3}$ and $c^{4}$. Again we choose an appropriate representative for $\kappa$ so that $r_{i}^{*}(\kappa)=b^{2}+b c^{2}$ for both $i$.

It is then clear that these three subgroups detect $H^{*}\left(D_{f_{2}} ; F_{2}\right)$.

Integrally, $H^{*}\left(D_{f_{2}} ; Z\right)=\left\langle e^{2}, f^{2}, h^{2}, e^{2} h+e h^{2}, e^{2} h+f^{2} h, \kappa\right\rangle$, with $\exp \left(\kappa^{n}\right)=2^{i+1}$, for each $n$. For the exponent of $\kappa^{n}$, we note that it cannot exceed $2^{i+1}$ as $D_{f_{2}}$ has a $Z_{2} \times Z_{2}$ subgroup. On the subgroup $\langle x, y z\rangle \cong Q_{2^{i+1}}, \kappa$ restricts to $\kappa \in H^{4}(Q ; Z)$ (this is clear by reducing mod-2) so the exponent must be at least $2^{i+1}$, and this subgroup will be necessary for detection.

The restriction maps are $r_{1}^{*}: e^{2} \mapsto 2^{i-1} b, f^{2} \mapsto 2^{i-1} b, h^{2} \mapsto c^{2} ; r_{2}^{*}: e^{2} \mapsto 0, f^{2} \mapsto$ $c^{2}, h^{2} \mapsto 2 b ; r_{3}^{*}: e^{2} \mapsto c^{2}, f^{2} \mapsto 0, h^{2} \mapsto 2 b ; \kappa \mapsto b^{2}+b c^{2}$ and the odd-degree elements are mapped to zero in each case. These are all determined by the BLES, except for $r^{*}(\kappa)$ which is found by reducing mod-2.

Let $r_{4}:\langle x, y z\rangle \cong Q \rightarrow D_{f_{2}}$ be inclusion. Then $r_{4}^{*}: e^{2} \mapsto a^{2}, f^{2} \mapsto b^{2}, h^{2} \mapsto$ $a^{2}+b^{2}, e^{2} h+e h^{2} \mapsto 0, e^{2} h+f^{2} h \mapsto 0, \kappa \mapsto \kappa$; all these are clear by reducing mod-2. Since $r_{4}^{*}$ picks up all integer multiples of $\kappa$ and this is the only higher torsion, these four subgroups detect $H^{*}\left(D_{f_{2}} ; Z\right)$ by Lemma 2 .

Let $w_{1}:(x, y, z) \mapsto(0,0,1), w_{2}:(x, y, z) \mapsto(0,1,1), w_{3}:(x, y, z) \mapsto(1,0,1)$, then we obtain

$H^{*}\left(D_{f_{2}} ; Z^{w_{1}}\right)=\left\langle h, e^{2}+e h, f^{2}+f h\right\rangle, H^{*}\left(D_{f_{2}} ; Z^{w_{2}}\right)=\langle e+f+h, e h, f h\rangle$, and $H^{*}\left(D_{f_{2}} ; Z^{w_{3}}\right)=\left\langle e+h, e h, f^{2}+f h\right\rangle$, all exponent two in each case. Since we have 
an injection $H^{*}\left(D_{f_{2}} ; Z^{w}\right) \rightarrow H^{*}\left(D_{f_{2}} ; F_{2}\right)$ for each $w$, the abelian subgroups will detect the twisted cohomology as they did in the mod-2 case.

$$
\text { A5. The group } \begin{aligned}
D_{2^{i+1}, f_{1}} & =\langle x, y, z| x^{2^{i}}=y^{2}=z^{2}=1, y x y^{-1}=x^{-1}, \\
z x z^{-1} & \left.=x x^{2^{i-1}}, z y z^{-1}=y\right\rangle
\end{aligned}
$$

We have $H^{*}\left(D_{f_{1}} ; F_{2}\right)=\left\langle e, f, h, e_{3}, \kappa\right\rangle / e f=e h^{2}=e e_{3}=0, \operatorname{dim}(\kappa)=4, \operatorname{dim}\left(e_{3}\right)$ $=3$, and on the spectral sequence $e_{3}$ represents $f \lambda^{2}$, where $\lambda$ is the generator of $H^{1}\left(Z_{2} ; F_{2}\right)$.

Let $r_{1}:\left\langle x^{2}, y, z\right\rangle \cong D_{2^{i}} \times Z_{2} \rightarrow D_{2^{i+1}, f_{1}}$ and $r_{2}:\left\langle x^{2}, x y, z\right\rangle \cong D_{2^{i}, f_{2}} \rightarrow D_{2^{i+1}, f_{1}}$ be inclusions. Then $r_{1}^{*}: e \mapsto 0, f \mapsto e+f, h \mapsto h, e_{3} \mapsto e h^{2}+e^{2} h+e w+f w$, $\kappa \mapsto w^{2}+w h^{2}+(e+f) w h ; r_{2}^{*}: e \mapsto e+f, f \mapsto 0, h \mapsto h, e_{3} \mapsto e h^{2}, \kappa \mapsto \kappa$. These two subgroups detect $H^{*}\left(D_{f_{1}} ; F_{2}\right)$, so it is eventually detected by abelian subgroups.

For $r_{2}^{*}\left(e_{3}\right)$ we need the Gysin sequence: $e h^{2} \in H^{3}\left(D_{f_{2}}\right)$ is in the kernel of transfer, hence in the image of $r_{2}^{*}$, and $e_{3}$ is the only thing available to hit it. In computing $r_{1}^{*}\left(e_{3}\right)$, the key calculation is $\operatorname{tr}(w)$ in the Gysin sequence, where $w \in H^{2}(D \times Z / 2)$. Using the detecting subgroups of $D \times Z / 2$, we obtain $c_{x}^{*}(w)=$ $w+h^{2}+e h+f h$, so $\operatorname{tr}(w)=h^{2}+f h$ plus possibly $e^{2}$ and $e h$. This implies $\operatorname{tr}((e+f) w)=f \operatorname{tr}(w)=f h^{2}+f^{2} h$, hence $e h^{2}+e^{2} h+e w+f w$ is in the kernel of transfer. Again, $e_{3}$ is the only element which can hit it. Similarly, the double coset formula gives us $\operatorname{tr}\left(w^{2}\right)=h^{4}+f^{2} h^{2}$ plus possibly $e^{4}$ and $e^{3} h$. The Gysin sequence shows that the $e^{4}$ and $e^{3} h$ terms are impossible, and also that $w^{2}+w h^{2}+(e+f) w h$ is in the kernel of transfer, hence the image of $r_{1}^{*}$. Classes representing $\kappa$ on the spectral sequence level are the only ones available to hit $w^{2}+w h^{2}+(e+f) w$, and we can choose $\kappa$ appropriately. Determining $r_{2}^{*}(\kappa)$ is similar.

Here we must be careful that we do not make incompatible choices when specifying the restrictions for $e_{3}$ and $\kappa$. It suffices to check that our two choices do not differ by elements which are not in the kernel of both restrictions. These elements are detected by the intersection $\left\langle x^{2}, z\right\rangle$ of the two subgroups. Checking to see that the usual intersection diagram commutes for both $\kappa$ and $e_{3}$ ensures that we do not have a contradiction.

We find $H^{*}\left(D_{f_{1}} ; Z\right)=\left\langle e^{2}, f^{2}, h^{2}, e^{2} h, f^{2} h+f h^{2}, \kappa, f^{2} e_{3}, h^{2} e_{3},\left(f^{2}+f h^{2}\right) e_{3}\right\rangle$, with $\exp \left(\kappa^{n}\right)=2^{i}$. The exponent of $\kappa^{n}$ is determined by injection of a maximal cyclic subgroup at the $E_{2}$ term of the BSS, and the ring structure is clear by reducing mod-2.

Let $r_{3}:\langle x\rangle \rightarrow D_{f_{1}}$ be inclusion. It is clear from the BLES that $r_{3}^{*}: e^{2} \mapsto$ $2^{i-1} b, f^{2} \mapsto 2^{i-1} b, h^{2} \mapsto 0,\left(f^{2} h+f h^{2}\right) e_{3} \mapsto 0, \kappa \mapsto b^{2}$, with all odd-degree classes going to zero. The restrictions $r_{1}^{*}$ and $r_{2}^{*}$ are almost entirely determined by reducing mod-2, since the integral cohomology of $D \times Z_{2}$ and $D_{f_{2}}$ has torsion greater than two only in degrees congruent to $0 \bmod 4$. This leaves only $r_{j}^{*}(\kappa)$, and even in this case the mod-2 reduction tells us that $r_{1}^{*}(\kappa)=w^{2}+w h^{2}+(e+f) w h$ and $r_{2}^{*}(\kappa)=\kappa$. Since $\langle x\rangle$ detects $\kappa^{n}$ and this is the only higher torsion, these three subgroups detect $H^{*}\left(D_{f_{1}} ; Z\right)$.

Let $w_{1}:(x, y, z) \mapsto(0,0,1)$ and $w_{2}:(x, y, z) \mapsto(0,1,1)$, then

$H^{*}\left(D_{f_{1}} ; Z^{w_{1}}\right)=\left\langle h, e^{2}+e h, f^{2}+f h, h e_{3},\left(f^{2}+f h\right) e_{3}\right\rangle$, all exponent two; $H^{*}\left(D_{f_{1}} ; Z^{w_{2}}\right)=\left\langle e+f+h, e h, f h,(f+h) e_{3}, f h e_{3}\right\rangle$, all exponent two.

Here again, $r_{1}^{*}$ and $r_{2}^{*}$ are determined by mod-2 reduction; $H^{*}\left(H ; Z^{\bar{w}}\right)$ is all 2torsion for both homomorphisms $w$ and for both subgroups. The restriction to the 
cyclic subgroup is completely given by the BLES. In the first twisting the restriction $r_{3}^{*}$ is $r_{3}^{*}: e^{2}+e h \mapsto 2^{i-1} b, f^{2}+f h \mapsto 2^{i-1} b, h e_{3} \mapsto 2^{i-1} b^{2}, \alpha \mapsto 0$, where $\alpha$ is any class of odd degree. In the second twisting the restriction is $r_{3}^{*}: e h \mapsto 2^{i-1} b, f h \mapsto$ $2^{i-1} b,(f+h) e_{3} \mapsto 2^{i-1} b^{2}, \alpha \mapsto 0$, for all $\alpha$ of odd degree.

Since there are no classes of high exponent, these three subgroups detect the integral cohomology since they detected mod-2. Furthermore, $\left(D_{f_{2}}, \bar{w}_{j}\right)$ and $(D \times$ $\left.Z_{2}, \bar{w}_{j}\right)$ are both detected by abelian subgroups for $j=1$ or 2 , so we in fact have abelian detection of these two $w$-basic groups.

$$
\begin{aligned}
& \text { A6. The Group } S D_{2^{i+1}, f_{3}}=\langle x, y, z| x^{2^{i}}=y^{2}=z^{2}=1, \\
& \left.y x y^{-1}=x^{-1} x^{2^{i-1}}, z x z^{-1}=x x^{2^{i-1}}, z y z^{-1}=y x^{2^{i-1}}\right\rangle
\end{aligned}
$$

We have $H^{*}\left(S D_{f_{3}} ; F_{2}\right)=\left\langle e, f, h, u_{5}, v_{5}, \kappa_{8}\right\rangle / e f=0, e^{3}=f h^{2}, e^{4}=f^{2} h^{2}=$ $e^{3} h^{2}=0, f u_{5}=e v_{5}=u_{5} v_{5}=e^{3} u_{5}=0, e^{2} u_{5}=h^{2} v_{5},\left(u_{5}\right)^{2}=e^{2} \kappa_{8}+e h^{4} u_{5},\left(v_{5}\right)^{2}=$ $f^{2} \kappa_{8}$, with $\operatorname{dim}\left(u_{5}\right)=\operatorname{dim}\left(v_{5}\right)=5, \operatorname{dim}\left(\kappa_{8}\right)=8, u_{5}$ represents $e \lambda^{4}$ and $v_{5}$ represents $f \lambda^{4}$ on the spectral sequence. This corrects A.1.5.6 in Appendix 1 of [4].

The elements $e^{3} h \cup \kappa_{8}^{n} \in H^{4+8 n}\left(S D_{f_{3}} ; F_{2}\right)$ are not detected by any proper subgroup. The groups $\left\langle x^{2}, y, z\right\rangle \cong Q_{2^{i}} \times Z_{2}$ and $\left\langle x^{2}, x y, z\right\rangle \cong D_{2^{i}, f_{2}}$, with restrictions $r_{1}$ and $r_{2}$, detect all but $e^{3} \kappa_{8}^{n}$ and $e^{3} h \kappa_{8}^{n}$. The maps $r_{1}^{*}$ and $r_{2}^{*}$ are determined using the Gysin sequence and the double coset formula and are as follows:

$$
r_{1}^{*}: e \mapsto a+b, f \mapsto 0, h \mapsto c, u_{5} \mapsto(a+b) \kappa+a c^{4}, v_{5} \mapsto a^{3} c^{2}, \kappa_{8} \mapsto \kappa^{2}+c^{4} \kappa ;
$$
$r_{2}^{*}: e \mapsto 0, f \mapsto e+f, h \mapsto h, u_{5} \mapsto e h^{4}, v_{5} \mapsto(e+f) \kappa, \kappa_{8} \mapsto \kappa^{2}$.

Both $r_{2}^{*}\left(u_{5}\right)$ and $r_{1}^{*}\left(v_{5}\right)$ are determined by the Gysin sequence: it is clear that both $e h^{4}$ and and $a^{3} c^{2}$ are in the kernel of transfer, and respectively $u_{5}$ and $v_{5}$ are the only possible classes to hit them.

Computing $r_{1}^{*}\left(u_{5}\right)$ is more delicate. First of all, to get sufficient information from the Gysin sequence, we need to know $\operatorname{tr}(\kappa)$, where $\kappa \in H^{4}(Q \times Z / 2)$. Exactness of the sequence forces $\operatorname{tr}(\kappa)$ to be $h^{4}$ plus possibly $e^{3} h, e^{2} h^{2}$, and $e h^{3}$. The double coset formula then implies $c_{x}^{*}(\kappa)=\kappa+c^{4}$ plus possibly $(a+b)^{2} c^{2}$ and $(a+b) c^{3}$. To narrow down the possiblities, we use the naturality of Steenrod squares. First, $S q^{1}\left(c_{x}^{*}(\kappa)\right)$ $=c_{x}^{*}\left(S q^{1}(\kappa)\right)=0$; thus, the $(a+b) c^{3}$ term is impossible. Second, $S q^{2}\left(c_{x}^{*}(\kappa)\right)=$ $c_{x}^{*}\left(S q^{2}(\kappa)\right)=0$; so, the $(a+b)^{2} c^{2}$ term is impossible.

We now have $\operatorname{tr}(\kappa)=h^{4}$ plus possibly $e^{3} h$. Regardless, $\operatorname{tr}((a+b) \kappa)=e \operatorname{tr}(\kappa)=$ $e h^{4}$, so that $(a+b) \kappa+a c^{4}$ is in the kernel of transfer. The only available classes to hit $(a+b) \kappa+a c^{4}$ are $u_{5}$ and $u_{5}+v_{5}$. Notice that here we cannot simply make a convenient choice for $u_{5}$ so that $r_{1}^{*}\left(u_{5}\right)$ is the desired element. Since either $u_{5}$ or $u_{5}+v_{5}$ could restrict to $(a+b) \kappa+a c^{4}$, by merely declaring $u_{5}$ to be the element which hits $(a+b) \kappa+a c^{4}$, we may end up with something which no longer represents $e \lambda^{4}$ on the spectral sequence. Hence, we must do a bit more work and determine whether $r_{1}^{*}\left(u_{5}\right)$ maps to $(a+b) \kappa+a c^{4}$ or to $(a+b) \kappa+a c^{4}+a^{3} c^{2}$.

With just the information we have so far, we know that $r_{1}^{*} \oplus r_{2}^{*}$ is injective on $H^{6}$. Using this, we check that $f u_{5}$ is zero, so $u_{5}$ is in the kernel of multiplication by $f$ and thus in the image of transfer. The Gysin sequence tells us that the element to hit $u_{5}$ must be $a \kappa$ plus possibly $c \kappa, a c^{4}, a^{2} c^{3}$. Let $\alpha$ be the class so that $\operatorname{tr}(\alpha)=u_{5}$, and the double coset formula gives us $r_{1}^{*}\left(u_{5}\right)=\alpha+c_{x}^{*}(\alpha)$. We already know that the left-hand side of the equation is $(a+b) \kappa+a c^{4}$ plus possibly $a^{3} c^{2}$; after writing out the right-hand side, we see that $\alpha$ must be $a \kappa+a c^{4}$. Now that $\alpha$ is determined, we apply the double coset formula again and see $r_{1}^{*}\left(u_{5}\right)=(a+b) \kappa+a c^{4}$.

The argument for $r_{2}^{*}\left(v_{5}\right)$ is almost identical. 
After having made the above computations, we proceed with the Gysin sequences and find immediately that $r_{1}^{*}\left(\kappa_{8}\right)=\kappa^{2}+c^{4} \kappa$, and that $r_{2}^{*}\left(\kappa_{8}\right)=\kappa^{2}$ plus possibly $e h^{3} \kappa$ and $h^{4} \kappa$. This can be quickly settled by considering the intersection of the two subgroups, $\left\langle x^{2}, z\right\rangle \cong Z / 2^{i-1} \times Z / 2$. If $r_{1}^{*}\left(\kappa_{8}\right)$ is $\kappa^{2}+c^{4} \kappa$, then on the intersection $\kappa_{8}$ restricts to $b^{4}+b^{2} c^{4}$. This forces $r_{2}^{*}\left(\kappa_{8}\right)$ to be $\kappa^{2}$.

With $S D_{f_{3}}$, as with $D_{f_{1}}$, we must take care that no contradictory choices are made in specifying $u_{5}, v_{5}$, and $\kappa_{8}$. The reader can easily check that no contradictions occur.

The terms $e^{3} \kappa_{8}^{n}$ are detected by the subgroup $\langle x z, y z\rangle \cong Q_{2^{i+1}}$. We compute this map by intersecting with the above two subgroups: $r_{3}^{*}: e \mapsto a, f \mapsto b, h \mapsto$ $b, u_{5} \mapsto a \kappa, v_{5} \mapsto b \kappa, \kappa_{8} \mapsto \kappa^{2}$.

We obtain $H^{*}\left(S D_{f_{3}} ; Z\right)=\left\langle e^{2}, f^{2}, h^{2}, e^{2} h+e h^{2}, f^{2} h+e^{3}, e^{3} h, e u_{5}, f v_{5}, e h u_{5}+\right.$ $\left.h^{2} u_{5}, f h v_{5}+h^{2} v_{5}, \kappa_{8}\right\rangle$, with $\exp \left(e^{3} h \kappa_{8}^{n}\right)=2^{i}$ and $\exp \left(\kappa_{8}\right)^{n}=2^{i+1}$. We would like to comment that this ring has eleven generators and at least fifty relations. Luckily, $H^{*}\left(S D_{f_{3}} ; Z\right)$ is detected by $\left\langle x^{2}, y, z\right\rangle,\left\langle x^{2}, x y, z\right\rangle$, and $\langle x z, y z\rangle$; one can use these subgroups to determine the ring structure.

We compute $r_{3}^{*}$ first. Since $H^{*}(Q ; Z)$ has torsion greater than two only in degrees congruent to $0 \bmod 4$, reducing mod 2 settles all cases but $e^{3} h$ and $\kappa_{8}$. The BLES handles both of these and we obtain $r_{3}^{*}\left(e^{3} h\right)=2 \kappa$ and $r_{3}^{*}\left(\kappa_{8}\right)=\kappa^{2}$.

Similarly, $r_{1}^{*}$ can be computed except for $e^{3} h$ by reducing $\bmod -2, r_{1}^{*}\left(\kappa_{8}\right)=$ $\kappa^{2}+c^{4} \kappa$. Reducing mod-2 tells us that $r_{1}^{*}\left(e^{3} h\right)=n \kappa$ for some $n$. As we already know $r_{3}^{*}$, we consider the intersection of $Q$ and $Q \times Z_{2}$ and the only possibility is then $r_{1}^{*}\left(e^{3} h\right)=2 \kappa \in H^{4}\left(Q_{2^{i}} \times Z_{2}\right)$.

For the last restriction mod-2 reduction settles every case except $e^{3} h$. This time we use the fact $r_{1}^{*}\left(e^{3} h\right)=2 \kappa$ and consider the intersection of $D_{f_{2}}$ and $Q \times Z_{2}$. Combined with the information from reducing mod-2, we obtain $r_{2}^{*}\left(e^{3} h\right)=2 \kappa \in$ $H^{4}\left(D_{f_{2}} ; Z\right)$.

To check that these three subgroups detect, notice that they detected $H^{j}\left(S D_{f_{3}} ; F_{2}\right)$ for all $j$ not congruent to $0 \bmod 4$. Since $H^{j}\left(S D_{f_{3}} ; Z\right)$ has no higher torsion in those degrees, by Lemma 2 they detect integrally. In $H^{4 n}\left(S D_{f_{3}} ; Z\right)$ we only need to worry about $e^{3} h \kappa_{8}^{n}, \kappa_{8}^{n}$ and their integer multiples, but all these classes are not in the kernel of $r_{3}^{*}$. Hence, $H^{*}\left(S D_{f_{3}} ; Z\right)$ is detected by $Q, Q \times Z_{2}$, and $D_{f_{2}}$.

Let $w_{1}:(x, y, z) \mapsto(0,0,1)$ and $w_{2}:(x, y, z) \mapsto(0,1,1) ; H^{*}\left(S D_{f_{3}} ; Z^{w_{1}}\right)=$ $\left\langle h, e h+e^{2}, f h+f^{2}, e u_{5}+h u_{5}, f v_{5}+h v_{5}, e h u_{5}, f h v_{5}\right\rangle$, all exponent two, and $H^{*}\left(S D_{f_{3}} ; Z^{w_{2}}\right)=\left\langle e+f+h, e h, f h, h u_{5}, h v_{5}, e^{2} u_{5}+e h u_{5}, f^{2} v_{5}+f h v_{5}\right\rangle$, all exponent two.

The module structure of $H^{*}\left(S D_{f_{3}} ; Z^{w}\right)$ in each case is determined by the injection to $H^{*}\left(S D_{f_{3}} ; F_{2}\right)$. Detection of all elements but $\kappa_{8}^{n} \cup e^{3} h$ follows from mod-2 detection.

We check to see if $e^{3} h \kappa_{8}^{n}$ is detected now. In the Bockstein spectral sequences, $e^{3} h$ is hit by $e^{3}$. The element $e^{3}$ in $H^{3}\left(S D_{f_{3}} ; F_{2}\right)$ is only detected by subgroups isomorphic to $Q$, namely $\langle x, y z\rangle$ and $\langle x z, y z\rangle$. We can see from the BLES that our only hope for detecting $e^{3} h \in H^{4}\left(S D_{f_{3}} ; Z^{w}\right)$ lies in the quaternionic subgroups. After applying the BLES, we discover $e^{3} h \in H^{4}\left(S D_{f_{3}} ; Z^{w_{2}}\right)$ restricts to $2^{i} \kappa$ in $H^{4}\left(\langle x, y z\rangle ; Z^{\bar{w}_{2}}\right)=H^{4}(\langle x, y z\rangle ; Z)$. However, $e^{3} h \in H^{4}\left(S D_{f_{3}} ; Z^{w_{1}}\right)$ is not detected by any subgroup, nor is $e^{3} h \kappa_{8}^{n} \in H^{8 n+4}\left(S D_{f_{3}} ; Z^{w_{1}}\right)$. We see in each case that $r^{*}\left(e^{3} h\right)=r^{*}\left(\beta^{w}\left(e^{3}\right)\right)=\beta^{\bar{w}}\left(r^{*}\left(e^{3}\right)\right)=\beta^{\bar{w}}\left(a^{3}\right)$, which is zero in each case, as we can recall from the computations for $Q$. 
Hence $S D_{f_{3}}$ is needed to detect $w$-basic groups in degrees congruent to 0 $\bmod 4$.

$$
\text { A7. The Group } W_{2^{i+1}}=\left\langle x, y \mid x^{2^{i}}=y^{2}=1, y x y^{-1}=x x^{2^{i-1}}\right\rangle
$$

We have $H^{*}\left(W ; F_{2}\right)=\left\langle e_{1}, c, e_{3}, \kappa\right\rangle /\left(e_{1}\right)^{2}=e_{1} \cup e_{3}=e_{1} c^{2}=\left(e_{3}\right)^{2}=0$, with $\operatorname{dim}\left(e_{1}\right)=\operatorname{dim}(c)=1, \operatorname{dim}\left(e_{3}\right)=3, \operatorname{dim}(\kappa)=4$. Here, $e_{1}$ is dual to $x$, and $c$ is dual to $y$.

Let $r_{1}:\langle x\rangle \rightarrow W$ and $r_{2}:\left\langle x^{2}, y\right\rangle \cong Z_{2^{i-1}} \times Z_{2} \rightarrow W$ be inclusions. Then we have $r_{1}^{*}: e_{1} \mapsto a, c \mapsto 0, e_{3} \mapsto a b, \kappa \mapsto b^{2}$, all are clear from the spectral sequence; and $r_{2}^{*}: e_{1} \mapsto 0, c \mapsto c, e_{3} \mapsto a c^{2}, \kappa \mapsto b^{2}+b c^{2}$. The last two computations are completely straightforward using the Gysin sequence and the double coset formula. These two subgroups detect all but $e_{1} c \cdot \kappa^{n}$, which is not detected by any proper subgroup.

We find $H^{*}(W ; Z)=\left\langle e_{1} c, c^{2}, e_{3} c^{2}, \kappa\right\rangle$, with $\exp \left(e_{1} c\right)=2^{i-1}$ and $\exp (\kappa)=2^{i}$. Here the exponent of $\kappa$ is found by injection of a maximal cyclic subgroup at the $E_{2}$ term of the BSS. Since $H_{1}(W ; Z)=W_{a b}=Z_{2^{i-1}} \times Z_{2}$, by universal coefficients, $\exp \left(e_{1} c\right)=2^{i-1}$. With $Z$ coefficients, the BLES determines both restrictions completely; $r_{1}^{*}\left(e_{1} c \kappa^{n}\right)=2 b^{2 n+1}, r_{1}^{*}\left(\kappa^{n}\right)=b^{2 n}, r_{1}^{*} \oplus r_{2}^{*}$ is injective.

Let $w_{1}: x \mapsto 0, y \mapsto 1$, and $w_{2}: x, y \mapsto 1$. Then $H^{*}\left(W ; Z^{w_{1}}\right)=\left\langle c, e_{1} c, e_{3} c\right\rangle$, $H^{*}\left(W ; Z^{w_{2}}\right)=\left\langle e_{1}+c, e_{1} c, e_{3} c\right\rangle$, all exponent two. Since both of these are all exponent two, any desired restriction map can be computed with the BLES. To determine detection, we need only check the classes which reduce mod-2 to undetected classes, i.e., $e_{1} c \kappa^{n}$ in each twisting. In $H^{*}\left(W ; Z^{w_{1}}\right)$ we are alright, as $r_{1}^{*}\left(e_{1} c \kappa^{n}\right)=2 b^{2 n+1}$. In $H^{*}\left(W ; Z^{w_{2}}\right)$ we need to use the other index-2 cyclic subgroup, $\langle x y\rangle$, and find $e_{1} c \kappa^{n}$ restricts to $2 b^{2 n+1}$. Hence, $W$ is integrally detected by abelians though it was not detected with $F_{2}$ coefficients.

\section{A8. The groups $G \times Z_{2}$, Where $G$ IS BasiC}

These groups are $w$-basic only for the homomorphism with $\operatorname{kernel}(w)=G$; the calculations are straightforward and are left for the reader. The only group of concern in terms of detection is $Q \times Z_{2}$, where the terms $a^{2} c^{2 n+1}$ and $a^{3} c^{2 n+1}$ are only detected by subgroups which are also isomorphic to $Q \times Z_{2}$. Eventually, we reach $Q_{8} \times Z_{2}$, where they are not detected at all. Thus we need $Q \times Z_{2}$ in all degrees to detect $w$-basic 2 -groups.

\section{ACKNOWLEDGMENT}

All research for this paper was done as a graduate student, and I would like to thank my thesis advisor, James F. Davis, for his patience and support.

\section{REFERENCES}

1. W. Browder, Torsion in $H$-spaces, Ann. of Math. 74 (1961), 24-61. MR 23:A2201

2. H. Cartan and S. Eilenberg, Homological algebra, Princeton Univ. Press, Princeton, NJ, 1956. MR 17:1040e

3. J. F. Davis, The rational symmetric signature of manifolds with finite fundamental group, submitted for publication.

4. J. F. Davis and R. J. Milgram, Semicharacteristics, bordism, and free group actions, Trans. Amer. Math. Soc. 312 (1989), 55-83. MR 90d:57036 
5. T. Diethelm, The mod-p cohomology rings of the non-abelian split metacyclic p-groups, Arch. Math. 44 (1985), 29-38. MR 86e:20057

6. L. Evens and S. Priddy, The cohomology of the semi-dihedral group, Contemp. Math. 37 (1985). MR 86h:20075

7. I. Hambleton, L. Taylor, and B. Williams, Detection theorems for K-theory and L-theory, J. Pure Appl. Algebra 63 (1990), 247-299. MR 91b:18015

8. I. Hambleton, R.J. Milgram, L. Taylor, and B. Williams, Surgery with finite fundamental group, Proc. London Math. Soc. 56 (1988), 349-379. MR 89c:57043

9. S. Mac Lane, Homology, Springer-Verlag, Berlin and New York, 1963. MR 30:1160

10. R. J. Milgram, Surgery with finite fundamental group, I and II, Pacific J. Math. 151 (1991), 65-150. MR 92j:57021a; MR 92j:57021b

11. H. Munkholm, Mod-2 cohomology of $D_{2^{n}}$ and its extensions by $Z_{2}$, Conference on Algebraic Topology, Univ. of Illinois Chicago Circle, 1968. MR 40:4374

12. E. K. Pedersen and A. Ranicki, Projective surgery theory, Topology 19 (1980), 239-254. MR 81i:57032

13. D. Rusin, The mod-2 cohomology of metacyclic 2-groups, J. Pure Appl. Algebra 44 (1987), 315-327. MR 88k:20076

14. The cohomology of the groups of order 32, Math. Comp. 53 (1989), 359-385. MR 89k:20078

15. Kernels of the restriction and inflation maps in group cohomology, J. Pure Appl. Algebra 79 (1992), 191-204. MR 93j:20101

16. L. Taylor and B. Williams, Surgery spaces: formulae and structure, Algebraic Topology (Waterloo 1978), Lecture Notes in Math., vol. 741, Springer-Verlag, Berlin and New York, 1979, pp. 170-195. MR 81k:57034

17. C.T.C. Wall, Formulae for surgery obstructions, Topology 25 (1976), 189-210. MR 54:11357

Department of Mathematics, Indiana University, Bloomington, Indiana 47405

E-mail address: klpearso@ucs.indiana.edu 